\section{Part III.II The Biochemical Approach to the Discovery of Antileishmanial Drugs}

\section{G M Coombs}

\section{Department of Zoology University of Glasgow Glasgow GL12 8QQ}

\section{Introduction}

There are many good reasons for studying the biochemistry of Leishmania. These parasites provide a marvellous model for investigating the process of cell differentiation, how it is regulated and what it entails. Leishmanias also provide a fascinating example of intracellular parasitism, and understanding the biochemical mechanisms that enable the parasites to survive within macrophages is a top priority. More generally, the complex Leishmania life cycles provide excellent models for studies aimed at elucidating how a single cell can adapt at the molecular level to a range of environments and how the variation in biochemistry is regulated. In this paper, however, I shall concentrate on yet another reason for studying leishmanial biochemistry the application of the knowledge gained about the parasite in the design of new antileishmanial agents, for which there is a great need.

\section{Biochemical Strategies}

The aims of the biochemist are basically quite simple. Discover differences between the parasite and the host and design drugs to exploit them. Easily said, but not so easily achieved. In recent years, however, large steps have been made to reaching the goal. Finding differences is really the simple part of the process and many are now known, notably in the areas of purine and pyrimidine metabolism, sterol content and biosynthesis, folate and polyamine metabolism, glutathione reductase and transsulphuration enzymes. The extent of the differences varies with the property, although in most cases we know insufficient details of the leishmanial component for a full assessment to be made. All the differences are potentially exploitable by drugs. As yet, however, we have not been clever enough knowingly to exploit any of them, although there have been several attempts and a few near misses. These include various inosine analogues, ketoconazole, difluormethlyornithine and inhibitors of dihydrofolate reductase. Studies are still in progress in most of these areas and so there is hope of future success.

Most of our current knowledge of leishmanial biochemistry has come from study of cultured promastigotes. But it is the amastigote that is responsible for the symptoms of the disease, and the "proboscis form" or "metacyclic" that probably is the infective agent. Relatively little is known about the biochemistry of these, but surely it is these that the biochemist should concentrate on - certainly if and where they differ from cultures? promastigotes.

Do the various morphological forms differ biochemi- 3 cally? Common sense tells us that they must, after all they have different structures and they live in distinct $O$ environments. Studies carried out in recent years have음 revealed a variety of differences between amastigotes $\stackrel{0}{?}$ and promastigotes; these are summarised in Table $1 . \vec{\Rightarrow}$ The situation becomes even more complicated when we $\stackrel{\vec{F}}{+}$ take into account that various promastigote forms exist; $\bar{c}$ we have shown that the infective-promastigotes (metacyclics) that occur in the stationary phase of cul- $\frac{\text { p }}{\vec{D}}$ tures differ at the molecular level from the log-phase $\mathbb{\infty}$ promastigotes (Mallinson \& Coombs, 1986). Clearly ® studies of log-phase promastigotes alone would lead to $\vec{\circ}$ some characteristics of the infective and intracellular stages being totally overlooked, characteristics that pre- $\vec{\omega}$ sumably play some vital role in causing the infection in man and so are drug targets of high potential.

Leishmanial proteinases provide perhaps the most clearcut example yet reported of a stage-specific feature $\frac{\dot{\omega}}{\omega}$ (Pupkis \& Coombs, 1984). Having discovered the N presence of a highly active, amastigote-specific pro- $\omega$ teinase, can we design drugs to exploit it? If suggested role of the enzyme in enabling the parasite $\bar{D}_{0}^{\omega}$ survive and grow in macrophages is true, then inhibition of it should lead to parasite destruction by the macrg $\overrightarrow{0}$ phage. We have shown that leupeptin has this effert against L.m. mexicana in macrophages in vitro; it $\frac{9}{65}$ hoped that such proteinase inhibitors may have useful ing vivo activity against this parasite. A second approach ${ }^{\circ} \vec{\omega}$ exploiting the amastigote proteinase is the use of the 10 to activate a so-called "pro-drug". Pro-drugs are cor pounds made up of two parts - the active drug that toxic to the target organism, and a second component that increases the specificity and so effectiveness of the drug. This may be achieved by targeting the drug to the $\frac{\mathscr{Q}}{\mathbb{Q}}$ site of the parasite - as is the rationale behind the use of $\varrho$ liposomes and antibodies bound to drugs. Another of $\overrightarrow{\overrightarrow{0}}$ our approaches with respect to the amastigote pro- 3 teinase is to combine a peptide group to the active drug such that the drug's biological activity is abolished, and would return only upon hydrolysis of the peptide bond $\bar{Z}$ and release of the free drug. In this way, the toxicity of the compound should be greatly reduced except in cells with the ability to split off the peptide group - which, of $\vec{\Phi}$ course, the amastigote has in abundance.

\section{The Future}

So far, I have considered Leishmania as though only one species exists. This would make it easier for the biochemist, although perhaps less challenging and excit- 0 ing. There are, of course, very many species of Leishmania and these differ to a greater or lesser extent $\frac{D}{0}$ in a variety of ways. Numbered amongst these are their biochemical features. For example, the occurrence of an $N$ amastigote-specific proteinase of high activity is an feature of $L$. mexicana but not other species; interest-N 
Table 1

Differences Between Leishmania M. Mexicana Stages

\begin{tabular}{|c|c|c|}
\hline & AMASTIGOTE & PROMASTIGOTE \\
\hline PROTEINASE & * & \\
\hline ARYLSULPHATASE & $*$ & \\
\hline RNASE & $*$ & \\
\hline DNASE & * & \\
\hline PEPTIDASES & & * \\
\hline GLUTAMATE DEHYDROGENASE & & $*$ \\
\hline SERINE SULPHYDRASE & & * \\
\hline PHOSPHOENOLPYRUVATE CARBOXYKINASE & $*$ & \\
\hline PYRUVATE KINASE & & * \\
\hline MALATEDEHYDROGENASE & * & \\
\hline OXYGEN REQUIREMENT & & * \\
\hline CARBON DIOXIDE REQUIREMENT & * & \\
\hline PURINE SALVAGE ENZYMES & & $*$ \\
\hline PYRIMIDINE BIOSYNTHESIS ENZYMES & & * \\
\hline PLASMA MEMBRANE STEROL & * & \\
\hline CARNITINE & * & \\
\hline FATTY ACID OXIDATION & $*$ & \\
\hline
\end{tabular}

ingly, the proteinase activities correlate well with the presence or absence of megasomes (Pupkis, Tetley \& Coombs, in press). These findings clearly emphasise the necessity of using the appropriate parasite form and species in future studies concerned with development of new antileishmanial agents.
REFERENCES

Mallinson D J and CoOmbs G H. (1986) IRCS Medical Science; in press.

Pupkis M F and Coombs G H. (1984) J Gen Microbiol; 130: 2375-2383.

Pupkis M F, Tetley L and Coombs G H. (1986) Exp Parasitol; in press. 